International Journal of Pure and Applied Mathematics

Volume 114 No. $4 \quad 2017,797-818$

ISSN: 1311-8080 (printed version); ISSN: 1314-3395 (on-line version)

url: http://www.ijpam.eu

doi: 10.12732/ijpam.v114i4.10

\title{
PRIMAL-DUAL ALGORITHMS FOR SEMIDEFINIT OPTIMIZATION PROBLEMS BASED ON GENERALIZED TRIGONOMETRIC
} BARRIER FUNCTION

\author{
M. El Ghami \\ Faculty of Education and Arts \\ Mathematics Section \\ Nord University-Nesna 8700, Nesna, NORWAY
}

\begin{abstract}
Recently, M. Bouafoa, et al. [5] (Journal of optimization Theory and Applications, August, 2016),investigated a new kernel function which differs from the self-regular kernel functions. The kernel function has a trigonometric Barrier Term. In this paper we generalize the analysis presented in the above paper for Semidefinit Optimization Problems (SDO). It is shown that the interior-point methods based on this function for large-update methods, the iteration bound is improved significantly. For small-update interior point methods the iteration bound is the best currently known bound for primal-dual interior point methods. The analysis for SDO deviates significantly from the analysis for linear optimization. Several new tools and techniques are derived in this paper.
\end{abstract}

AMS Subject Classification: 90C22, 90C31

Key Words: interior-point, kernel function, primal-dual method semidefinite optimization, large update, small update

\section{Introduction}

We consider the standard semidefinite optimization problem (SDO)

$$
(S D P) \quad p^{*}=\inf _{X}\left\{\operatorname{Tr}(C X): \operatorname{Tr}\left(A_{i} X\right)=b_{i}(1 \leq i \leq m), X \succeq 0\right\},
$$

and its dual problem $(S D D)$

Received: January 24, 2017

Revised: $\quad$ March 17, 2017

Published: $\quad$ June 7, 2017
(C) 2017 Academic Publications, Ltd. url: www.acadpubl.eu 


$$
(S D D) \quad d^{*}=\sup _{y, S}\left\{b^{T} y: \sum_{i=1}^{m} y_{i} A_{i}+S=C, S \succeq 0\right\},
$$

where $C$ and $A_{i}$ are symmetric $n \times n$ matrices, $b, y \in \mathbf{R}^{m}$, and $X \succeq 0$ means that $X$ is symmetric positive semidefinite and $\operatorname{Tr}(A)$ denotes the trace of $A$ (i.e., the sum of its diagonal elements). Without loss of generality the matrices $A_{i}$ are assumed to be linearly independent. Recall that for any two $n \times n$ matrices, $A$ and $B$ their natural inner product is given by

$$
\operatorname{Tr}\left(A^{T} B\right)=\sum_{i=1}^{n} \sum_{j=1}^{n} A_{i j} B_{i j} .
$$

IPMs provide a powerful approach for solving SDO problems. A comprehensive list of publications on SDO can be found in the SDO homepage maintained by Alizadeh [1]. Pioneering works are due to Alizadeh $[1,2]$ and Nesterov et al[11]. Most IPMs for SDO can be viewed as natural extensions of IPMs for linear optimization (LO), and have similar polynomial complexity results. However, to obtain valid search directions is much more difficult than in the LO case. In the sequel we describe how the usual search directions are obtained for primal-dual methods for solving SDO problems. Our aim is to show that the kernel-function-based approach that we presented for LO in [7] can be generalized and applied also to SDO problems.

\subsection{Classical search direction}

We assume that (SDP) and (SDD) satisfy the interior-point condition (IPC), i.e., there exists $X^{0} \succ 0$ and $\left(y^{0}, S^{0}\right)$ with $S^{0} \succ 0$ such that $X^{0}$ is feasible for (SDP) and $\left(y^{0}, S^{0}\right)$ is feasible for (SDD). Moreover, we may assume that $X^{0}=S^{0}=E$, where $E$ is the $n \times n$ identity matrix [12]. Assuming the IPC, one can write the optimality conditions for the primal-dual pair of problems as follows.

$$
\begin{aligned}
\operatorname{Tr}\left(A_{i} X\right) & =b_{i}, \quad i=1, \ldots, m \\
\sum_{i=1}^{m} y_{i} A_{i}+S & =C \\
X S & =0 \\
X, S & \succeq 0 .
\end{aligned}
$$

The basic idea of primal-dual IPMs is to replace the complementarity condition 
$X S=0$ by the parameterized equation

$$
X S=\mu E ; \quad X, S \succ 0
$$

where $\mu>0$. The resulting system has a unique solution for each $\mu>0$. This solution is denoted by $(X(\mu), y(\mu), S(\mu))$ for each $\mu>0 ; X(\mu)$ is called the $\mu$-center of $(S D P)$ and $(y(\mu), S(\mu))$ is the $\mu$-center of $(S D D)$. The set of $\mu$ centers (with $\mu>0$ ) defines a homotopy path, which is called the central path of $(S D P)$ and $(S D D)[12,13]$. The principal idea of IPMs is to follow this central path and approach the optimal set as $\mu$ goes to zero. Newton's method amounts to linearizing the system (1), thus yielding the following system of equations.

$$
\begin{aligned}
\operatorname{Tr}\left(A_{i} \Delta X\right) & =0, \quad i=1, \ldots, m \\
\sum_{i=1}^{m} \Delta y_{i} A_{i}+\Delta S & =0 \\
X \Delta S+\Delta X S & =\mu E-X S .
\end{aligned}
$$

This so-called Newton system has a unique solution $(\Delta X, \Delta y, \Delta S)$. Note that $\Delta S$ is symmetric, due to the second equation in (2). However, a crucial point is that $\Delta X$ may be not symmetric. Many researchers have proposed various ways of 'symmetrizing' the third equation in the Newton system so that the new system has a unique symmetric solution. All these proposals can be described by using a symmetric nonsingular scaling matrix $P$ and by replacing $(2)$ by the system

$$
\begin{aligned}
\operatorname{Tr}\left(A_{i} \Delta X\right) & =0, \quad i=1, \ldots, m \\
\sum_{i=1}^{m} \Delta y_{i} A_{i}+\Delta S & =0 \\
\Delta X+P \Delta S P^{T} & =\mu S^{-1}-X
\end{aligned}
$$

Now $\Delta X$ is automatically a symmetric matrix.

\subsection{Nesterov-Todd direction}

In this paper we consider the symmetrization schema of Nesterov-Todd [14]. So we use

$$
P=X^{\frac{1}{2}}\left(X^{\frac{1}{2}} S X^{\frac{1}{2}}\right)^{-\frac{1}{2}} X^{\frac{1}{2}}=S^{-\frac{1}{2}}\left(S^{\frac{1}{2}} X S^{\frac{1}{2}}\right)^{\frac{1}{2}} S^{-\frac{1}{2}}
$$


where the last equality can be easily verified. Let $D=P^{\frac{1}{2}}$, where $P^{\frac{1}{2}}$ denotes the symmetric square root of $P$. Now, the matrix $D$ can be used to scale $X$ and $S$ to the same matrix $V$, namely $[12,15]$ :

$$
V:=\frac{1}{\sqrt{\mu}} D^{-1} X D^{-1}=\frac{1}{\sqrt{\mu}} D S D .
$$

Obviously the matrices $D$ and $V$ are symmetric, and positive definite. Let us further define

$$
\bar{A}_{i}:=\frac{1}{\sqrt{\mu}} D A_{i} D, \quad i=1,2, \ldots, m ;
$$

and

$$
D_{X}:=\frac{1}{\sqrt{\mu}} D^{-1} \Delta X D^{-1} ; \quad D_{S}:=\frac{1}{\sqrt{\mu}} D \Delta S D .
$$

We refer to $D_{X}$ and $D_{S}$ as the scaled search directions. Now (3) can be rewritten as follows:

$$
\begin{aligned}
\operatorname{Tr}\left(\bar{A}_{i} D_{X}\right) & =0, \quad i=1, \ldots, m . \\
\sum_{i=1}^{m} \Delta y_{i} \bar{A}_{i}+D_{S} & =0 \\
D_{X}+D_{S} & =V^{-1}-V .
\end{aligned}
$$

In the sequel, we use the following notational conventions. Throughout this paper, $\|\cdot\|$ denotes the 2 -norm of a vector. The nonnegative and the positive orthants are denoted as $\mathbf{R}_{+}^{n}$ and int $\mathbf{R}_{+}^{n}$, respectively, and $\mathbf{S}^{\mathbf{n}}, \mathbf{S}_{+}^{\mathbf{n}}$, and int $\mathbf{S}_{+}^{n}$ denote the cone of symmetric, symmetric positive semidefinite and symmetric positive definite $n \times n$ matrices, respectively. For any $V \in \mathbf{S}^{\mathbf{n}}$, we denote by $\lambda(V)$ the vector of eigenvalues of $V$ arranged in increasing order, $\lambda_{1}(V) \leq \lambda_{2}(V) \leq$ $, \ldots, \lambda_{n}(V)$. For any square matrix $A$, we denote by $\eta_{1}(A) \leq \eta_{2}(A) \leq, \ldots, \leq$ $\eta_{n}(A)$ the singular values of $A$; if $A$ is symmetric, then one has $\eta_{i}(A)=\left|\lambda_{i}(A)\right|$, $i=1,2, \ldots, n$. If $z \in \mathbf{R}^{n}$ and $f: \mathbf{R} \rightarrow \mathbf{R}$, then $f(z)$ denotes the vector in $\mathbf{R}^{n}$ whose $i$-th component is $f\left(z_{i}\right)$, with $1 \leq i \leq n$, and if $D$ is a diagonal matrix, then $f(D)$ denotes the diagonal matrix with $f\left(D_{i i}\right)$ as $i$ diagonal component. For $X \in S^{n}, X=Q^{-1} D Q$, where $Q$ is orthogonal, and $D$ a diagonal matrix, $f(X)=Q^{-1} f(D) Q$. Finally if $v$ is a vector, $\operatorname{diag}(v)$ denotes the diagonal matrix with the diagonal elements $v_{i}$.

\section{New search direction}

In this section we introduce the new search direction. But we start with the definition of a matrix function $[16,17]$. 
Definition 1. Let $X$ be a symmetric matrix, and let

$$
X=Q_{X}^{-1} \operatorname{diag}\left(\lambda_{1}(X), \lambda_{2}(X), \ldots, \lambda_{n}(X)\right) Q_{X}
$$

be an eigenvalue decomposition of $X$, where $\lambda_{i}(X), 1 \leq i \leq n$ denotes the $i$ th eigenvalue of $X$, and $Q_{X}$ is orthogonal. If $\psi(t)$ is any univariate function whose domain contains $\left\{\lambda_{i}(X) ; 1 \leq i \leq n\right\}$ then the matrix function $\psi(X)$ is defined by

$$
\psi(X)=Q_{X}^{-1} \operatorname{diag}\left(\psi\left(\lambda_{1}(X)\right), \psi\left(\lambda_{2}(X)\right), \ldots, \psi\left(\lambda_{n}(X)\right)\right) Q_{X} .
$$

and the scalar function $\Psi(X)$ is defined as follows [13]:

$$
\Psi(X):=\sum_{i=1}^{n} \psi\left(\lambda_{i}(X)\right)=\operatorname{Tr}(\psi(X)) .
$$

The univariate function $\psi$ is called the kernel function of the scalar function $\Psi$.

In this paper, when we use the function $\psi(\cdot)$ and its first three derivatives $\psi^{\prime}(\cdot), \psi^{\prime \prime}(\cdot)$, and $\psi^{\prime \prime \prime}(\cdot)$ without any specification, it denotes a matrix function if the argument is a matrix and a univariate function (from $\mathbf{R}$ to $\mathbf{R}$ ) if the argument is in $\mathbf{R}$.

Analogous to the case of LO, the kernel-function-based approach to SDO is obtained by modifying Nesterov-Todd direction [13].

The observation underlying our approach is that the right-hand side $V^{-1}-V$ in the third equation of (6) is precisely $-\psi^{\prime}(V)$ if $\psi(t)=\left(t^{2}-1\right) / 2-\log t$, the latter being the kernel function of the well-known logarithmic barrier function. Note that this kernel function is strictly convex and nonnegative, whereas its domain contains all positive reals and it vanishes at 1 . As we will now show any continuously differentiable kernel function $\psi(t)$ with these properties gives rise to a primal-dual algorithm for SDO.

Given such a kernel function $\psi(t)$ we replace the right-hand side $V^{-1}-V$ in the third equation of $(6)$ by $-\psi^{\prime}(V)$, with $\psi^{\prime}(V)$ defined according to Definition 1. Thus we use the following system to define the (scaled) search directions $D_{X}$ an $D_{S}$ :

$$
\begin{aligned}
\operatorname{Tr}\left(\bar{A}_{i} D_{X}\right) & =0, \quad i=1, \ldots, m . \\
\sum_{i=1}^{m} \Delta y_{i} \bar{A}_{i}+D_{S} & =0 \\
D_{X}+D_{S} & =-\psi^{\prime}(V) .
\end{aligned}
$$


Having $D_{X}$ and $D_{S}, \triangle X$ and $\triangle S$ can be calculated from (5). Due to the orthogonality of $\triangle X$ and $\triangle S$, it is trivial to see that $D_{X} \perp D_{S}$, and so

$$
\operatorname{Tr}\left(D_{X} D_{S}\right)=\operatorname{Tr}\left(D_{S} D_{X}\right)=0
$$

The algorithm considered in this paper is described in Figure 1. The inner

\section{Generic Primal-Dual Algorithm for SDO}

\section{Input:}

a kernel function $\psi(t)$;

a threshold parameter $\tau \geq 1$;

an accuracy parameter $\epsilon>0$;

a barrier update parameter $\theta, 0<\theta<1$;

$$
\begin{aligned}
& \text { begin } \\
& X:=E ; S:=E ; \mu:=1 ; V:=E \text {; } \\
& \text { while } n \mu \geq \epsilon \text { do } \\
& \text { begin } \\
& \mu:=(1-\theta) \mu ; \\
& V:=\frac{V}{\sqrt{1-\theta}} ; \\
& \text { while } \Psi(V) \geq \tau \text { do } \\
& \text { begin } \\
& X=X+\alpha \triangle X \\
& y=y+\alpha \Delta y \\
& S=S+\alpha \triangle S \text {; } \\
& \text { Compute } V \text { from (4); } \\
& \text { end } \\
& \text { end }
\end{aligned}
$$

Find search directions by solving system (8);

Figure 1: Generic primal-dual interior-point algorithm for SDO.

while loop in the algorithm is called inner iteration and the outer while loop outer iteration. So each outer iteration consists of an update of the barrier parameter and a sequence of one or more inner iterations. Note that by using 
the embedding strategy [12], we can initialize the algorithm with $X=S=E$. Since then $X S=\mu E$ for $\mu=1$ it follows from (4) that $V=E$ at the start of the algorithm, whence $\Psi(V)=0$. We then decrease $\mu$ to $\mu:=(1-\theta) \mu$, for some $\theta \in(0,1)$. In general this will increase the value of $\Psi(V)$ above the threshold value $\tau$. To get this value smaller again, and coming closer to the current $\mu$-center, we solve the scaled search directions from (8), and unscale these directions by using (5). By choosing an appropriate step size $\alpha$, we move along the search direction, and construct a new triple $\left(X_{+}, y_{+}, S_{+}\right)$with

$$
X_{+}=X+\alpha \triangle X \quad y_{+}=y+\alpha \Delta y \quad S_{+}=S+\alpha \triangle S
$$

If necessary, we repeat the procedure until we find iterates such that $\Psi(V)$ no longer exceed the threshold value $\tau$, which means that the iterates are in a small enough neighborhood of $(X(\mu), y(\mu), S(\mu))$. Then $\mu$ is again reduced by the factor $1-\theta$ and we apply the same procedure targeting at the new $\mu$-centers. This process is repeated until $\mu$ is small enough, i.e. until $n \mu \leq \epsilon$. At this stage we have found an $\epsilon$-solution of $(S D P)$ and $(S D D)$. Just as in the $L O$ case, the parameters $\tau, \theta$, and the step size $\alpha$ should be chosen in such a way that the algorithm is 'optimized' in the sense that the number of iterations required by the algorithm is as small as possible. Obviously, the resulting iteration bound will depend on the kernel function underlying the algorithm, and our main task becomes to find a kernel function that minimizes the iteration bound.

The rest of the paper is organized as follows. In Section 3 we introduce the kernel function $\psi(t)$ considered in this paper and discuss some of its properties that are needed in the analysis of the corresponding algorithm. In Section 4 we derive the properties of the barrier function $\Psi(V)$. The step size $\alpha$ and the resulting decrease of the barrier function are discussed in Section 5. The total iteration bound of the algorithm and the complexity results are derived in Section 6. Finally, some concluding remarks follow in Section 7.

\section{Our kernel function and some of its properties}

Recently in [5] investigated new kernel functions with trigonometric barrier for linear optimization. The extension to $P_{*}(\kappa)$-linear complementarity problem was also presented in [10]. The obtained complexity for large-update improve significantly the complexity obtained in $[6,7]$. In this paper we consider kernel functions of the form 


$$
\psi(t)=\frac{t^{2}-1}{2}+\frac{4}{p \pi}\left(\tan ^{p}(h(t))-1\right),
$$

with $h(t)=\frac{\pi}{2 t+2}$, and to show that the interior-point methods for SDO based on these function have favorable complexity results.

Note that the growth term of our kernel function is quadratic. However, this function (11) deviates from all other kernel functions since its barrier term is trigonometric as $\frac{4}{p \pi}\left(\tan ^{p}\left(\frac{\pi}{2 t+2}\right)-1\right)$. In order to study the new kernel function, several new arguments had to be developed for the analysis.

This section is started by technical lemma, and then some properties of the new kernel function introduced in this paper are derived.

\subsection{Some technical results}

In the analysis of the algorithm based on $\psi(t)$ we need its first three derivatives. These are given by

$$
\begin{aligned}
\psi^{\prime}(t) & =t+\frac{4 h^{\prime}(t)}{\pi} \sec ^{2}(h(t))\left(\tan ^{p-1}(h(t))\right), \\
\psi^{\prime \prime}(t) & =1+\frac{4}{\pi} \sec ^{2}(h(t)) g(t) . \\
\psi^{\prime \prime \prime}(t) & =\frac{4}{\pi} \sec ^{2}(h(t))\left(k(t) h^{\prime}(t)^{3}+r(t) h^{\prime \prime}(t) h^{\prime}(t)\right) h^{\prime \prime \prime}(t) \\
& +\left(\tan ^{p-1}(h(t)) h^{\prime \prime \prime}(t)\right),
\end{aligned}
$$

with

$$
\begin{aligned}
g(t) & :=\left((p-1) \tan ^{p-2}(h(t))+(p+1) \tan ^{p}(h(t))\right) h^{\prime}(t)^{2} \\
& +h^{\prime \prime}(t) \tan ^{p-1}(h(t)), \\
k(t) & :=(p-1)(p-2) \tan ^{p-3}(h(t))+2 p^{2} \tan ^{p-1}(h(t)) \\
& +(p+1)(p+2) \tan ^{p+1}(h(t)),
\end{aligned}
$$

and

$$
r(t):=3(p-1) \tan ^{p-2}(h(t))+3(p+1) \tan ^{p}(h(t)),
$$

and the first three derivatives of $h$ are given by

$$
h^{\prime}(t)=\frac{-\pi}{2(t+1)^{2}} ; \quad h^{\prime \prime}(t)=\frac{\pi}{(t+1)^{3}} ; \quad h^{\prime \prime \prime}(t)=\frac{-3 \pi}{(t+1)^{4}}
$$


The next lemma serves to prove that the new kernel function (11) is eligible. Then,

Lemma 2 (Lemma 3.2 in [5]). Let $\psi$ be as defined in (11) and $t>0$.

$$
\begin{aligned}
\psi^{\prime \prime}(t) & >1, \\
t \psi^{\prime \prime}(t)+\psi^{\prime}(t) & >0, \\
t \psi^{\prime \prime}(t)-\psi^{\prime}(t) & >0, \\
\text { and } \psi^{\prime \prime \prime}(t) & <0 .
\end{aligned}
$$

It follows that $\psi(1)=\psi^{\prime}(1)=0$ and $\psi^{\prime \prime}(t) \geq 0$, proving that $\psi$ is defined by $\psi^{\prime \prime}(t)$.

$$
\psi(t)=\int_{1}^{t} \int_{1}^{\xi} \psi^{\prime \prime}(\zeta) d \zeta d \xi .
$$

The second property (17-b) in Lemma 2 is related to Definition 2.1.1 and Lemma 2.1.2 in [13]. This property is equivalent to convexity of the composed function $z \mapsto \psi\left(e^{z}\right)$ and this holds if and only if $\psi\left(\sqrt{t_{1} t_{2}}\right) \leq \frac{1}{2}\left(\psi\left(t_{1}\right)+\psi\left(t_{2}\right)\right)$ for any $t_{1}, t_{2} \geq 0$. Following [3], we therefore say that $\psi$ is exponentially convex, or shortly, e-convex, whenever $t>0$.

Lemma 3. Let $\psi$ be as defined in (11), one has

$$
\psi(t)<\frac{1}{2} \psi^{\prime \prime}(1)(t-1)^{2}, \quad \text { if } t>1 .
$$

Proof. By Taylor's theorem and $\psi(1)=\psi^{\prime}(1)=0$, we obtain

$$
\psi(t)=\frac{1}{2} \psi^{\prime \prime}(1)(t-1)^{2}+\frac{1}{6} \psi^{\prime \prime \prime}(\xi)(\xi-1)^{3},
$$

where $1<\xi<t$ if $t>1$. Since $\psi^{\prime \prime \prime}(\xi)<0$, the lemma follows.

Lemma 4. Let $\psi$ be as defined in (11), one has

$$
t \psi^{\prime}(t) \geq \psi(t), \text { if } t \geq 1 \text {. }
$$

Proof. Defining $g(t):=t \psi^{\prime}(t)-\psi(t)$ one has $g(1)=0$ and $g^{\prime}(t)=t \psi^{\prime \prime}(t) \geq$ 0 . Hence $g(t) \geq 0$ and the lemma follows.

At some places below we apply the function $\Psi$ to a positive vector $v$. The interpretation of $\Psi(v)$ is compatible with Definition 1 when identifying the vector $v$ with its diagonal matrix $\operatorname{diag}(v)$. When applying $\Psi$ to this matrix we obtain

$$
\Psi(v)=\sum_{i=1}^{n} \psi\left(v_{i}\right), \quad v \in \operatorname{int} \mathbf{R}_{+}^{n} .
$$




\section{Properties of $\Psi(V)$ and $\delta(V)$}

In this section we extend Theorem 4.9 in [4] to the cone of positive definite matrices. The next theorem gives a lower bound on the norm-based proximity measure $\delta(V)$, defined by

$$
\delta(V)=\frac{1}{2}\left\|\psi^{\prime}(V)\right\|=\frac{1}{2} \sqrt{\sum_{i=1}^{n} \psi^{\prime}\left(\lambda_{i}(V)\right)^{2}}=\frac{1}{2}\left\|D_{X}+D_{S}\right\|,
$$

in terms of $\Psi(V)$. Since $\Psi(V)$ is strictly convex and attains its minimal value zero at $V=E$, we have

$$
\Psi(V)=0 \quad \Leftrightarrow \quad \delta(V)=0 \quad \Leftrightarrow \quad V=E .
$$

We denote by $\varrho:[0, \infty) \rightarrow[1, \infty)$ the inverse function of $\psi(t)$ for $t \geq 1$. In other words

$$
s=\psi(t) \quad \Leftrightarrow \quad t=\varrho(s), \quad t \geq 1,
$$

Theorem 5. Let $\varrho$ be as defined in (20). Then

$$
\delta(V) \geq \frac{1}{2} \psi^{\prime}(\varrho(\Psi(V))) .
$$

Proof. If $V=E$ then $\delta(V)=\Psi(V)=0$. Since $\varrho(0)=1$ and $\psi^{\prime}(1)=0$, the inequality holds with equality if $V=E$. Otherwise, by the definitions of $\delta(V)$ in (19) and $\Psi(V)$ in (7), we have $\delta(V)>0$ and $\Psi(V)>0$. Let $v_{i}:=\lambda_{i}(V)$, $1 \leq i \leq n$. Then $v>0$ and

$$
\delta(V)=\frac{1}{2} \sqrt{\sum_{i=1}^{n} \psi^{\prime}\left(\lambda_{i}(V)\right)^{2}}=\frac{1}{2} \sqrt{\sum_{i=1}^{n} \psi^{\prime}\left(v_{i}\right)^{2}} .
$$

Since $\psi(t)$ satisfies (17-d) we may apply Theorem 4.9 in [4] to the vector $v$. This gives

$$
\delta(V) \geq \frac{1}{2} \psi^{\prime}\left(\varrho\left(\sum_{i=1}^{n} \psi\left(v_{i}\right)\right)\right) .
$$

Since

$$
\sum_{i=1}^{n} \psi\left(v_{i}\right)=\sum_{i=1}^{n} \psi\left(\lambda_{i}(V)\right)=\Psi(V),
$$

the proof of the theorem is complete. 
Lemma 6. If $\Psi(V) \geq 1$, then

$$
\delta(V) \geq \frac{1}{6} \Psi(V)^{\frac{1}{2}}
$$

Proof. The proof of this lemma uses Theorem 5 and Lemma 4. Putting $s=\Psi(V)$, we obtain from Theorem 5 that

$$
\delta(V) \geq \frac{1}{2} \psi^{\prime}(\varrho(s)) .
$$

Putting $t=\varrho(s)$, we have by $(20)$,

$$
\psi(t)=\frac{t^{2}-1}{2}+\frac{4}{p \pi}\left(\tan ^{p}(h(t))-1\right)=s, \quad \text { with } \quad h(t)=\frac{\pi}{2 t+2}, \quad t \geq 1 .
$$

We derive an upper bound for $t$, as this suffices for our goal. One has from (18) and $\psi^{\prime \prime}(t) \geq 1$,

$$
s=\psi(t)=\int_{1}^{t} \int_{1}^{\xi} \psi^{\prime \prime}(\zeta) d \zeta d \xi \geq \int_{1}^{t} \int_{1}^{\xi} d \zeta d \xi=\frac{1}{2}(t-1)^{2}
$$

which implies

$$
t=\varrho(s) \leq 1+\sqrt{2 s}
$$

Assuming $s \geq 1$, we get $t=\varrho(s) \leq \sqrt{s}+\sqrt{2 s} \leq 3 s^{\frac{1}{2}}$. Now applying Lemma 4 we may write

$$
\delta(V) \geq \frac{1}{2} \psi^{\prime}(\varrho(s)) \geq \frac{\psi(\varrho(s))}{2 \varrho(s)}=\frac{s}{2 \varrho(s)} \geq \frac{1}{6} s^{\frac{1}{2}}=\frac{1}{6} \Psi(V)^{\frac{1}{2}} .
$$

This proves the lemma.

Note that since $\tau \geq 1$ we have at the start of each inner iteration that $\Psi(V) \geq 1$. Substitution in (21) gives

$$
\delta(V) \geq \frac{1}{6}
$$

\section{Analysis of the algorithm}

In the analysis of the algorithm the concept of exponential convexity $[4,8]$ is again a crucial ingredient. In this section we derive a default value for the step 
size and we obtain an upper bound for the decrease in $\Psi(V)$ during a Newton step.

\subsection{Three technical lemmas}

The next lemma is cited from [16, Lemma 3.3.14 (c)].

Lemma 7. Let $A, B \in \mathbf{S}^{n}$ be two nonsingular matrices and $f(t)$ be given real-valued function such that $f\left(e^{t}\right)$ is a convex function. One has

$$
\sum_{i=1}^{n} f\left(\eta_{i}(A B)\right) \leq \sum_{i=1}^{n} f\left(\eta_{i}(A) \eta_{i}(B)\right)
$$

where $\eta_{i}(A)$, and $\eta_{i}(B) i=1,2, \ldots, n$ denote the singular values of $A$ and $B$ respectively

Lemma 8. Let $A, A+B \in \mathbf{S}_{+}^{\mathbf{n}}$, then one has

$$
\lambda_{i}(A+B) \geq \lambda_{1}-\left|\lambda_{n}(B)\right|, \quad i=1,2, \ldots, n .
$$

Proof. It is obvious that $\lambda_{i}(A+B) \geq \lambda_{1}(A+B)$. By the Rayleigh-Ritz theorem (see [18]), there exists a nonzero $X_{0} \in \mathbf{R}^{n}$, such that

$$
\lambda_{1}(A+B)=\frac{X_{0}^{T}(A+B) X_{0}}{X_{0}^{T} X_{0}}=\frac{X_{0}^{T} A X_{0}}{X_{0}^{T} X_{0}}+\frac{X_{0}^{T} B X_{0}}{X_{0}^{T} X_{0}} .
$$

We therefore may write

$$
\begin{aligned}
\lambda_{1}(A+B) & \geq \frac{X_{0}^{T} A X_{0}}{X_{0}^{T} X_{0}}-\left|\frac{X_{0}^{T} B X_{0}}{X_{0}^{T} X_{0}}\right| \\
& \geq \min _{X \neq 0} \frac{X^{T} A X}{X^{T} X}-\max _{X \neq 0}\left|\frac{X^{T} B X}{X^{T} X}\right|=\lambda_{1}-\left|\lambda_{n}(B)\right| .
\end{aligned}
$$

This completes the proof of the lemma.

A consequence of condition (17-b) is that any eligible kernel function is exponentially convex [13, Eq. (2.10)]:

$$
\psi\left(\sqrt{t_{1} t_{2}}\right) \leq \frac{1}{2}\left(\psi\left(t_{1}\right)+\psi\left(t_{2}\right)\right), \quad \forall t_{1}>0, \forall t_{2}>0
$$

This implies the following Lemma, which is crucial for our purpose.

Lemma 9. Let $V_{1}$ and $V_{2}$ be two symmetric positive definite matrices, then

$$
\Psi\left(\left(V_{1}^{\frac{1}{2}} V_{2} V_{1}^{\frac{1}{2}}\right)^{\frac{1}{2}}\right) \leq \frac{1}{2}\left(\Psi\left(V_{1}\right)+\Psi\left(V_{2}\right)\right), \quad \forall V_{1} \succ 0, \forall V_{2} \succ 0 .
$$


Proof. For any nonsingular matrix $U \in \mathbf{S}^{n}$, we have

$$
\eta_{i}(U)=\left(\lambda_{i}\left(U^{T} U\right)\right)^{\frac{1}{2}}=\left(\lambda_{i}\left(U U^{T}\right)\right)^{\frac{1}{2}}, \quad i=1,2, \ldots, n
$$

Taking $U=V_{1}^{\frac{1}{2}} V_{2}^{\frac{1}{2}}$, we may write

$$
\eta_{i}\left(V_{1}^{\frac{1}{2}} V_{2}^{\frac{1}{2}}\right)=\left(\lambda_{i}\left(V_{1}^{\frac{1}{2}} V_{2} V_{1}^{\frac{1}{2}}\right)\right)^{\frac{1}{2}}=\lambda_{i}\left(\left(V_{2}^{\frac{1}{2}} V_{1} V_{2}^{\frac{1}{2}}\right)\right)^{\frac{1}{2}}, \quad i=1,2, \ldots, n .
$$

Since $V_{1}$ and $V_{2}$ are symmetric positive definite, using Lemma 7 one has

$$
\Psi\left(\left(V_{1}^{\frac{1}{2}} V_{2} V_{1}^{\frac{1}{2}}\right)^{\frac{1}{2}}\right)=\sum_{i=1}^{n} \psi\left(\eta_{i}\left(V_{1}^{\frac{1}{2}} V_{2}^{\frac{1}{2}}\right)\right) \leq \sum_{i=1}^{n} \psi\left(\eta_{i}\left(V_{1}^{\frac{1}{2}}\right) \eta_{i}\left(V_{2}^{\frac{1}{2}}\right)\right)
$$

Since $\eta_{1}\left(V_{1}^{\frac{1}{2}}\right), \eta_{1}\left(V_{2}^{\frac{1}{2}}\right)>0$ we may use that $\psi(t)$ satisfies $(17-\mathrm{b})$ for $t>0$. Using (24), hence we obtain

$$
\begin{aligned}
\Psi\left(\left(V_{1}^{\frac{1}{2}} V_{2} V_{1}^{\frac{1}{2}}\right)^{\frac{1}{2}}\right) & \leq \frac{1}{2} \sum_{i=1}^{n}\left(\psi\left(\eta_{i}^{2}\left(V_{1}^{\frac{1}{2}}\right)\right)+\psi\left(\eta_{i}^{2}\left(V_{2}^{\frac{1}{2}}\right)\right)\right) \\
& =\frac{1}{2} \sum_{i=1}^{n}\left(\psi\left(\lambda_{i}\left(V_{1}\right)\right)+\psi\left(\lambda_{i}\left(V_{2}\right)\right)\right)=\frac{1}{2}\left(\Psi\left(V_{1}\right)+\Psi\left(V_{2}\right)\right) .
\end{aligned}
$$

This completes the proof.

\subsection{The decrease of the proximity in the inner iteration}

In this subsection we are going to compute a default value for the step size $\alpha$ in order to yield a new triple $\left(X_{+}, y_{+}, S_{+}\right)$as defined in (10). After a damped step, using (5) we have

$$
\begin{aligned}
X_{+} & =X+\alpha \Delta X=X+\alpha \sqrt{\mu} D D_{X} D=\sqrt{\mu} D\left(V+\alpha D_{X}\right) D \\
y_{+} & =y+\alpha \Delta y \\
S_{+} & =S+\alpha \Delta S=X+\alpha \sqrt{\mu} D^{-1} D_{S} D^{1}=\sqrt{\mu} D^{-1}\left(V+\alpha D_{S}\right) D^{-1} .
\end{aligned}
$$

Denoting the matrix $V$ after the step as $V_{+}$, we have

$$
V_{+}=\frac{1}{\sqrt{\mu}}\left(D^{-1} X_{+} S_{+} D\right)^{\frac{1}{2}}
$$


Note that $V_{+}^{2}$ is unitarily similar to the matrix $\frac{1}{\mu} X_{+}^{\frac{1}{2}} S_{+} X_{+}^{\frac{1}{2}}$ and hence also to

$$
\left(V+\alpha D_{X}\right)^{\frac{1}{2}}\left(V+\alpha D_{S}\right)\left(V+\alpha D_{X}\right)^{\frac{1}{2}} .
$$

This implies that the eigenvalues of $V_{+}$are the same as those of the matrix

$$
\tilde{V}_{+}:=\left(\left(V+\alpha D_{X}\right)^{\frac{1}{2}}\left(V+\alpha D_{S}\right)\left(V+\alpha D_{X}\right)^{\frac{1}{2}}\right)^{\frac{1}{2}} .
$$

The definition of $\Psi(V)$ implies that its value depends only on the eigenvalues of $V$. Hence we have

$$
\Psi\left(\tilde{V}_{+}\right)=\Psi\left(V_{+}\right) .
$$

Our aim is to find $\alpha$ such that the decrement

$$
f(\alpha):=\Psi\left(V_{+}\right)-\Psi(V)=\Psi\left(\tilde{V}_{+}\right)-\Psi(V),
$$

is as small as possible. Due to Lemma 9, it follows that

$$
\begin{aligned}
\Psi\left(\tilde{V}_{+}\right) & =\Psi\left(\left(\left(V+\alpha D_{X}\right)^{\frac{1}{2}}\left(V+\alpha D_{S}\right)\left(V+\alpha D_{X}\right)^{\frac{1}{2}}\right)^{\frac{1}{2}}\right) \\
& \leq \frac{1}{2}\left[\Psi\left(V+\alpha D_{X}\right)+\Psi\left(V+\alpha D_{S}\right)\right] .
\end{aligned}
$$

From the definition $(25)$ of $f(\alpha)$, we now have $f(\alpha) \leq f_{1}(\alpha)$, where

$$
f_{1}(\alpha):=\frac{1}{2}\left[\Psi\left(V+\alpha D_{X}\right)+\Psi\left(V+\alpha D_{S}\right)\right]-\Psi(V) .
$$

Note that $f_{1}(\alpha)$ is convex in $\alpha$, since $\Psi$ is convex. Obviously, $f(0)=f_{1}(0)=0$.

Taking the derivative with respect to $\alpha$, we get

$$
f_{1}^{\prime}(\alpha)=\frac{1}{2} \operatorname{Tr}\left(\psi^{\prime}\left(V+\alpha D_{X}\right) D_{X}+\psi^{\prime}\left(V+\alpha D_{S}\right) D_{S}\right) .
$$

Using the last equality in (8) and also (19), this gives

$$
f_{1}^{\prime}(0)=\frac{1}{2} \operatorname{Tr}\left(\psi^{\prime}(V)\left(D_{X}+D_{S}\right)\right)=-\frac{1}{2} \operatorname{Tr}\left(\psi^{\prime}(V)^{2}\right)=-2 \delta(V)^{2} .
$$

Differentiating once more, we obtain

$$
f_{1}^{\prime \prime}(\alpha)=\frac{1}{2} \operatorname{Tr}\left(\psi^{\prime \prime}\left(V+\alpha D_{X}\right) D_{X}^{2}+\psi^{\prime \prime}\left(V+\alpha D_{S}\right) D_{S}^{2}\right) .
$$

In the sequel we use the following notation:

$$
\lambda_{1}:=\min \left(\lambda_{i}(V)\right), \quad \delta:=\delta(V) .
$$

Lemma 10. One has

$$
f_{1}^{\prime \prime}(\alpha) \leq 2 \delta^{2} \psi^{\prime \prime}\left(\lambda_{1}-2 \alpha \delta\right) .
$$


Proof. The last equality in (8) and (19) imply that $\left\|D_{X}+D_{S}\right\|^{2}=\left\|D_{X}\right\|^{2}+$ $\left\|D_{S}\right\|^{2}=4 \delta^{2}$. Thus we have $\left|\lambda_{n}\left(D_{X}\right)\right| \leq 2 \delta$ and $\left|\lambda_{n}\left(D_{S}\right)\right| \leq 2 \delta$. Using Lemma 8 and $V+\alpha D_{X} \succeq 0$, As a consequence we have, for each $i$,

$$
\begin{aligned}
\lambda_{i}\left(V+\alpha D_{X}\right) & \geq \lambda_{1}-\alpha\left|\lambda_{n}\left(D_{X}\right)\right| \geq \lambda_{1}-2 \alpha \delta, \\
\lambda_{i}\left(V+\alpha D_{S}\right) & \geq \lambda_{1}-\alpha\left|\lambda_{n}\left(D_{S}\right)\right| \geq \lambda_{1}-2 \alpha \delta .
\end{aligned}
$$

Due to $(17-\mathrm{d}), \psi^{\prime \prime}$ is monotonically decreasing. So the above inequalities imply that

$$
\psi^{\prime \prime}\left(\lambda_{i}\left(V+\alpha D_{X}\right)\right) \leq \psi^{\prime \prime}\left(\lambda_{1}-2 \alpha \delta\right), \quad \psi^{\prime \prime}\left(\lambda_{i}\left(V+\alpha D_{S}\right)\right) \leq \psi^{\prime \prime}\left(\lambda_{1}-2 \alpha \delta\right) .
$$

Substitution into (26) gives

$$
f_{1}^{\prime \prime}(\alpha) \leq \frac{1}{2} \psi^{\prime \prime}\left(\lambda_{1}-2 \alpha \delta\right) \operatorname{Tr}\left(D_{X}^{2}+D_{S}^{2}\right)=\frac{1}{2} \psi^{\prime \prime}\left(\lambda_{1}-2 \alpha \delta\right)\left(\left\|D_{X}\right\|^{2}+\left\|D_{S}\right\|^{2}\right) .
$$

Now, using that $D_{X}$ and $D_{S}$ are orthogonal, by (9), and also $\left\|D_{X}+D_{S}\right\|^{2}=$ $4 \delta^{2}$, by (19), we obtain

$$
f_{1}^{\prime \prime}(\alpha) \leq 2 \delta^{2} \psi^{\prime \prime}\left(\lambda_{1}(V)-2 \alpha \delta\right) .
$$

This proves the lemma.

Using the notation $v_{i}=\lambda_{i}(V), 1 \leq i \leq n$, again, we have

$$
f_{1}^{\prime \prime}(\alpha) \leq 2 \delta^{2} \psi^{\prime \prime}\left(v_{1}-2 \alpha \delta\right),
$$

which is exactly the same inequality as Lemma 3.1 in [7]. This means that our analysis closely resembles the analysis of the LO case in [7]. From this stage on we can apply similar arguments as in the LO case. In particular, the following two lemmas can be stated without proof.

Lemma 11. [Lemmas 3.3 and 3.4 in [9]] Let $\rho$ be the inverse function of $-\frac{1}{2} \psi^{\prime}(t)$ for $t \in(0,1]$. Then the largest value of the step size $\alpha$ satisfying $(27)$ is given by

$$
\hat{\alpha}:=\frac{1}{2 \delta}[\rho(\delta)-\rho(2 \delta)] .
$$

Moreover

$$
\hat{\alpha} \geq \frac{1}{\psi^{\prime \prime}(\rho(2 \delta))} .
$$

For future use we define

$$
\widetilde{\alpha}:=\frac{1}{\psi^{\prime \prime}(\rho(2 \delta))} .
$$

By Lemma 11 this step size satisfies (27). 
Lemma 12. If the step size $\alpha$ is such that $\alpha \leq \hat{\alpha}$ then

$$
f(\alpha) \leq-\alpha \delta^{2}
$$

Using the above lemmas from [7] we proceed as follows.

Theorem 13. Let $\rho$ be as defined in Lemma 11 and $\widetilde{\alpha}$ as in (28) and $\Psi(v) \geq 1$. Then

$$
f(\tilde{\alpha}) \leq-\frac{\delta^{2}}{\psi^{\prime \prime}(\rho(2 \delta))} \leq-\frac{\delta^{\frac{p}{1+p}}}{1320 p}
$$

Proof. Since $\widetilde{\alpha} \leq \hat{\alpha}$, Lemma 12 gives $f(\widetilde{\alpha}) \leq-\widetilde{\alpha} \delta^{2}$, where $\widetilde{\alpha}=\frac{1}{\psi^{\prime \prime}(\rho(2 \delta))}$ as defined in (28). Thus the first inequality follows.

To obtain the inverse function $t=\rho(s)$ of $-\frac{1}{2} \psi^{\prime}(t)$ for $t \in(0,1]$, we need to solve $t$ from the equation

$$
\begin{aligned}
& -\left(t+\frac{4 h^{\prime}(t)}{\pi} \sec ^{2}(h(t))\left(\tan ^{p-1}(h(t))\right)\right) \\
= & \left(-t+\frac{4 h^{\prime}(t)}{\pi} \csc ^{2}(h(t))\left(\tan ^{p+1}(h(t))\right)\right)=2 s .
\end{aligned}
$$

This implies,

$$
\csc ^{2}(h(t))\left(\tan ^{p+1}(h(t))\right)=\frac{-\pi}{4 h^{\prime}(t)}(2 s+t) .
$$

For $t \leq 1$, we get $\frac{2 \pi(t+1)^{2}}{4 \pi}(2 s+t) \leq 2(2 s+1)$. Hence, putting $t=\rho(2 \delta)$, which is equivalent to $4 \delta=-\psi^{\prime}(t)$. Using that $\sin ^{2}(h(t)) \leq 1$ we get

$$
\tan (h(t)) \leq(8 \delta+2)^{\frac{1}{1+p}}
$$

Since $\sec ^{2}(h(t))=1+\tan ^{2}(h(t))$, By $(29)$, thus we have $\tan ^{2}(h(t)) \leq(8 \delta+2)^{\frac{2}{1+p}}$, $\tan ^{p-2}(h(t)) \leq(8 \delta+2)^{\frac{p-2}{1+p}}, \tan ^{p-1}(h(t)) \leq(8 \delta+2)^{\frac{p-1}{1+p}}$ and $\tan ^{p}(h(t)) \leq$ $(8 \delta+2)^{\frac{p}{1+p}}$. Since $h^{\prime \prime}(t)=\frac{8 \pi}{8(t+1)^{3}} \leq \frac{3 \pi}{4}$, and $h^{\prime}(t)^{2}=\frac{4 \pi^{2}}{16(2 t+1)^{4}} \leq \frac{\pi^{2}}{4}$ for all $0 \leq t \leq 1$, and using also $(8 \delta+2) \geq 1$ this implies

$$
\psi^{\prime \prime}(t) \leq\left(1+\frac{4}{\pi} 2\left(2 p \frac{\pi^{2}}{4}+\pi\right)\right)(8 \delta+2)^{\frac{p+2}{1+p}}=(9+4 p \pi)(8 \delta+2)^{\frac{p+2}{1+p}}
$$


By (28), thus we have

$$
\begin{aligned}
\widetilde{\alpha} & =\frac{1}{\psi^{\prime \prime}(\rho(2 \delta))} \\
& \geq \frac{1}{(9+4 p \pi)(8 \delta+2)^{\frac{p+2}{1+p}}} .
\end{aligned}
$$

Also using (23) (i.e., $6 \delta \geq 1$ ) and $p \geq 2$ we get,

$$
\begin{aligned}
\widetilde{\alpha} & \geq \frac{1}{(9+4 p \pi)(8 \delta+12 \delta)^{\frac{p+2}{1+p}}} \\
& =\frac{1}{(9+4 p \pi)(20 \delta)^{\frac{p+2}{1+p}}} \geq \frac{1}{1320 p \delta^{\frac{p+2}{1+p}}} .
\end{aligned}
$$

Hence

$$
f(\widetilde{\alpha}) \leq-\frac{\delta^{2}}{1320 p \delta^{\frac{p+2}{1+p}}}=-\frac{\delta^{\frac{p}{1+p}}}{1320 p}
$$

Thus the theorem follows.

Substitution in (21) gives

$$
\begin{aligned}
f(\tilde{\alpha}) \leq-\frac{\delta^{\frac{p}{1+p}}}{1320 p} & \leq-\frac{\Psi^{\frac{p}{2(1+p)}}}{1320 p(6)^{\frac{p}{1+p}}} \\
& \leq-\frac{\Psi^{\frac{p}{2(1+p)}}}{7920 p} .
\end{aligned}
$$

\subsection{A uniform upper bound for $\Psi$}

In this subsection we extend Theorem 3.2 in [4] to the cone of positive definite matrices. As we will see the proof of the next theorem easily follows from Theorem 3.2 in [4].

Theorem 14. Let $\varrho$ be as defined in (20). Then for any positive vector $v$ and any $\beta>1$ we have:

$$
\Psi(\beta V) \leq n \psi\left(\beta \varrho\left(\frac{\Psi(V)}{n}\right)\right) .
$$

Proof. Let $v_{i}:=\lambda_{i}(V), 1 \leq i \leq n$. Then $v>0$ and

$$
\Psi(\beta V)=\sum_{i=1}^{n} \psi\left(\lambda_{i}(\beta V)\right)=\sum_{i=1}^{n} \psi\left(\beta \lambda_{i}(V)\right)=\sum_{i=1}^{n} \psi\left(\beta v_{i}\right)=\Psi(\beta v) .
$$


Due to the fact that $\psi(t)$ satisfies (17-c), at this stage we may use Theorem 3.2 in [4], which gives

$$
\Psi(\beta v) \leq n \psi\left(\beta \varrho\left(\frac{\Psi(v)}{n}\right)\right)
$$

Since

$$
\Psi(v)=\sum_{i=1}^{n} \psi\left(v_{i}\right)=\sum_{i=1}^{n} \psi\left(\lambda_{i}(V)\right)=\Psi(V),
$$

the theorem follows.

Before the update of $\mu$ we have $\Psi(V) \leq \tau$, and after the update of $\mu$ to $(1-\theta) \mu$ we have $V_{+}=\frac{V}{\sqrt{1-\theta}}$. Application of Theorem 14, with $\beta=\frac{1}{\sqrt{1-\theta}}$, yields that

$$
\Psi\left(V_{+}\right) \leq n \psi\left(\frac{\varrho\left(\frac{\tau}{n}\right)}{\sqrt{1-\theta}}\right)
$$

Therefore we define

$$
L=L(n, \theta, \tau):=n \psi\left(\frac{\varrho\left(\frac{\tau}{n}\right)}{\sqrt{1-\theta}}\right)
$$

In the sequel the value $L(n, \theta, \tau)$ is simply denoted as $L$. A crucial (but trivial) observation is that during the course of the algorithm the value of $\Psi(V)$ will never exceed $L$, because during the inner iterations the value of $\Psi$ always decreases.

\section{Complexity}

We are now ready to derive the iteration bounds for large-update methods. An upper bound for the total number of (inner) iterations is obtained by multiplying an upper bound for the number of inner iterations between two successive updates of $\mu$ by the number of barrier parameter updates. The last number is bounded above by (cf. [19, Lemma II.17, page 116])

$$
\frac{1}{\theta} \log \frac{n}{\epsilon}
$$

To obtain an upper bound $K$ for the number of inner iterations between two successive updates we need a few more technical lemmas. 
The following lemma is taken from Proposition 1.3.2 in [13]. Its relevance is due to the fact that the barrier function values between two successive updates of $\mu$ yield a decreasing sequence of positive numbers. We will denote this sequence as $\Psi_{0}, \Psi_{1}, \ldots$

Lemma 15. Let $t_{0}, t_{1}, \cdots, t_{K}$ be a sequence of positive numbers such that

$$
t_{k+1} \leq t_{k}-\kappa t_{k}^{1-\gamma}, \quad k=0,1, \cdots, K-1,
$$

where $\kappa>0$ and $0<\gamma \leq 1$. Then $K \leq\left\lfloor\frac{t_{0}^{\gamma}}{\kappa \gamma}\right\rfloor$.

Lemma 16. If $K$ denotes the number of inner iterations between two successive updates of $\mu$, then

$$
K \leq 7920 p \Psi_{0}^{\frac{2+p}{2(1+p)}}
$$

Proof. The definition of $K$ implies $\Psi_{K-1}>\tau$ and, according to Theorem $13, \Psi_{K} \leq \tau$ and

$$
\Psi_{k+1} \leq \Psi_{k}-\kappa\left(\Psi_{k}\right)^{1-\gamma}, \quad k=0,1, \cdots, K-1
$$

with $\kappa=\frac{1}{7920 p}$ and $\gamma=\frac{2+p}{2(1+p)}$. Application of Lemma 15, with $t_{k}=\Psi_{k}$ yields the desired inequality.

Using $\psi_{0} \leq L$, where the number $L$ is as given in (30), and Lemma 16 we obtain the following upper bound on the total number of iterations:

$$
\frac{7920 p L^{\frac{2+p}{2(1+p)}}}{\theta} \log \frac{n}{\epsilon}
$$

\subsection{Large-update}

We just established that (31) is an upper bound for the total number of iterations, using

$$
\psi(t)=\frac{t^{2}-1}{2}+\frac{4}{p \pi}\left(\tan ^{p}\left(\frac{\pi}{2 t+2}\right)-1\right), \text { for } t \geq 1, \quad p \geq 2
$$

and (22), by substitution in (30) we obtain

$$
L \leq n \frac{\left(\frac{\varrho\left(\frac{\tau}{n}\right)}{\sqrt{1-\theta}}\right)^{2}-1}{2} \leq \frac{n}{2(1-\theta)}\left(\theta+2 \sqrt{2 \frac{\tau}{n}}+\frac{2 \tau}{n}\right)
$$




$$
=\frac{(\theta n+2 \sqrt{2 \tau n}+2 \tau)}{2(1-\theta)} .
$$

Using (31), thus the total number of iterations is bounded above by

$$
\frac{K}{\theta} \log \frac{n}{\epsilon} \leq \frac{7920 p}{\theta\left(2(1-\theta)^{\frac{2+p}{2(1+p)}}\right)}(\theta n+2 \sqrt{2 \tau n}+2 \tau)^{\frac{2+p}{2(1+p)}} \log \frac{n}{\epsilon} .
$$

A large-update methods uses $\tau=O(n)$ and $\theta=\Theta(1)$. The right-hand side expression is then $O\left(p n^{\frac{2+p}{2(1+p)}} \log \frac{n}{\epsilon}\right)$, as easily may be verified.

\subsection{Small-update}

For small-update methods one has $\tau=O(1)$ and $\theta=\Theta\left(\frac{1}{\sqrt{n}}\right)$. Using Lemma 3 , with $\psi^{\prime \prime}(1)=\frac{p \pi+8}{4}$, we then obtain

$$
L \leq \frac{n(p \pi+8)}{8}\left(\frac{\rho\left(\frac{\tau}{n}\right)}{\sqrt{1-\theta}}-1\right)^{2}
$$

Using (22), then

$$
L \leq \frac{n(p \pi+8)}{8}\left(\frac{1+\sqrt{\frac{2 \tau}{n}}}{\sqrt{1-\theta}}-1\right)
$$

Using $1-\sqrt{1-\theta}=\frac{\theta}{1+\sqrt{1-\theta}} \leq \theta$, this leads to $L \leq \frac{(p \pi+8)}{8(1-\theta)}(\theta \sqrt{n}+\sqrt{2 \tau})^{2}$. We conclude that the total number of iterations is bounded above by

$$
\frac{K}{\theta} \log \frac{n}{\epsilon} \leq \frac{7920(p \pi+8)^{\frac{2+p}{2(1+p)}}}{\theta(8(1-\theta))^{\frac{2+p}{2(1+p)}}}(\theta \sqrt{n}+\sqrt{2 \tau})^{\frac{2+p}{1+p}} \log \frac{n}{\epsilon}
$$

Thus the right-hand side expression is then $O\left(\sqrt{n} \log \frac{n}{\epsilon}\right)$.

\section{Concluding Remarks}

In this paper we extended the results obtained for kernel-function-based IPMs in [5] for LO to semidefinite optimization problems. The analysis in this paper is new and different from the one using for $L O$. Several new tools and techniques are derived in this paper. The proposed function has a trigonometric barrier term but the function is not logarithmic and not self- regular. 
For this parametric kernel function, we have shown that the best result of iteration bounds for large-update methods and small-update can be achieved, namely $O\left(\log n \sqrt{n} \log \frac{n}{\epsilon}\right)$, for large-update and $O\left(\sqrt{n} \log \frac{n}{\epsilon}\right)$ for small-update methods.

\section{References}

[1] F. Alizadeh, Combinatorial Optimization with Interior Point Methods and Semi-Definite Matrices, PhD thesis, University of Minnesota, Minneapolis, Minnesota, USA (1991).

[2] F. Alizadeh, Interior point methods in semidefinite programming with applications to combinatorial optimization. SIAM Journal on Optimization, 5 No 1 (1995), 13-51, doi: $10.1137 / 0805002$.

[3] Y. Q. Bai, M. El Ghami, C. Roos, A new efficient large-update primal-dual interiorpoint method based on a finite barrier. SIAM Journal on Optimization, 13 No 3 (2003), 766-782, doi: 10.1137/S1052623401398132.

[4] Y. Q. Bai, M. El Ghami, C. Roos, A comparative study of kernel functions for primaldual interior-point algorithms in linear optimization. SIAM Journal on Optimization, 15 No 1 (2004), 101-128, doi: 10.1137/S1052623403423114.

[5] M. Bouafia, D. Benterki, A. Yassine, An efficient primal-dual interior point method for linear programing problems based on a new kernel function with a trigonometric barrier term. Journal of Optimization Theory and Applications, 170 No 2 (2016), 528-545, doi: 10.1007/s10957-016-0895-0.

[6] X.Z. Cai, G.Q. Wang, M. El Ghami, Y.J. Yue, Complexity analysis of primal-dual interior-point methods for linear optimization based on a parametric kernel function with a trigonometric barrier term. Abstr. Appl. Anal. (2014), 710158, doi: $10.1155 / 2014 / 710158$.

[7] M. El Ghami, Z.A. Guennoun, S. Bouali, T. Steihaug, Primal-Dual Interior-Point Methods for Linear Optimization Based on a Kernel Function with Trigonometric Barrier Term. Journal of Computational and Applied Mathematics, 236 No 15 (2012), 3613-3623, doi: 10.1016/j.cam.2011.05.036.

[8] M. El Ghami, A kernel function approach for interior point methods: Analysis and Implementation. LAP Lambert Academic Publishing, Germany, NP. 168, ISBN-NR: 978-38443-3333-6 (2011).

[9] M. El Ghami, C. Roos, Generic Primal-dual Interior Point Methods Based on a New Kernel Function. International Journal RAIRO-Operations Research, 42 No 2 (2008), 199-213, doi: 10.1051/ro:2008009.

[10] M. El Ghami, G.Q. Wang, Interior-point methods for $P_{*}(\kappa)$-linear complementarity problem based on generalized trigonometric barrier functio. International Journal of Applied Mathematics, 30 No 1 (2017), 11-33, doi: 10.12732/ijam.v30i1.2.

[11] Y.E. Nesterov, A.S. Nemirovskii, Interior Point Polynomial Methods in Convex Programming: Theory and Algorithms, SIAM, Philadelphia, USA (1993).

[12] E. de Klerk, Aspects of Semidefintie Programming, volume 65 of Applied Optimization, Kluwer Academic Publishers, Dordrecht, The Netherlands (2002). 
[13] J. Peng, C. Roos, T. Terlaky, Self-Regularity: A New Paradigm for Primal-Dual InteriorPoint Algorithms, Princeton University Press (2002).

[14] Y.E. Nesterov, M.J., Todd, Self-scaled barriers and interior-point methods for convex programming. Mathematics of Operations Research, 22 No 1 (1997), 1-42.

[15] J.F. Sturm, S. Zhang, Symmetric primal-dual path following algorithms for semidefinite programming. Applied Numirical Mathematics, 29 (1999), 301-315, doi: 10.1016/S01689274(98)00099-3.

[16] R.A. Horn, C.R. Johnson, Matrix Analysis, Cambridge University Press, Cambridge, UK (1985).

[17] W. Rudin, Principles of Mathematical Analysis, Mac-Graw Hill Book Company, New York (1978).

[18] R.A. Horn, C.R. Johnson, Topics in Matrix Analysis, Cambridge University Press (1991).

[19] C. Roos, T. Terlaky, J.-Ph. Vial, Theory and Algorithms for Linear Optimization. An Interior-Point Approach, Springer Science (2005). 